\title{
Der kosmopolitische Büchersammler. Zu August Wilhelm Schlegels Verzeichniß meiner Bücher im December 1811
}

Im Dezember 1811, wohl bei einem seiner letzten Besuche in Coppet vor seiner Flucht mit Madame de Staël, inventarisierte August Wilhelm Schlegel seine 1083 Titel umfassende Bibliothek. Dieses Verzeichniß meiner Bücher im December $1811^{1}$ ist der Schlegel-Forschung längst bekannt und von ihr teilweise ausgewertet. ${ }^{2}$ Dennoch sind immer noch bedeutende Aufschlüsse daraus zu gewinnen: über Schlegels Wissen, über seinen kosmopolitischen Dialog mit Nachbarfächern, etwa durch Übersetzung, und wie sich der gelehrte Horizont generell ins Interdisziplinäre erweiterte, was für die romantische Generation im Allgemeinen und für Schlegel im Besonderen charakteristisch ist.

Wer von Schlegels Bücherverzeichnis eine fein säuberlich geordnete Sammlung etwa nach den Richtlinien seines Göttinger Lehrers und Mentors Christoph Gottlob Heyne erwartet, wird schwer enttäuscht. Die Sammlung ist nämlich nicht nach Wissensbereichen oder Sachgebieten geordnet, sondern nach Buchformaten: Folio, Quarto, Octavo, Duodecimo. Schlegel wusste offenbar, wo ein jedes Buch zu finden war; der moderne Forscher muss hingegen mühsam suchen. Es ist nur begrenzt zulässig, aus dem Bücherbesitz Rückschlüsse über Schlegels eigenes Interesse und seine Veranlagung zu gewinnen, wenngleich etwa das auffällige Fehlen ganzer Bereiche der englischen Literatur auf ein Desinteresse oder das Vorhandensein von Texten zur Mathematik und zum Schachspiel auf ein Faible für diese Themen schließen ließen.

Erst der so genannte Heberle-Katalog von $1845,{ }^{3}$ der Auktionskatalog von Schlegels nachgelassener Bibliothek, hat, durch die sachlichen Überlegungen des Bücherverkaufs motiviert, seine Büchersammlung nach Sachgebieten ge-

1 Sächsische Landesbibliothek - Staats- und Universitätsbibliothek Dresden (SLUB). Mscr. Dresd. e. 90. XV. - Ich danke Claudia Bamberg und Cornelia Bögel sehr herzlich für ihre freundliche Hilfe beim vorliegenden Beitrag.

2 Vgl. Körner, Josef: Die Bibliothek August Wilhelm von Schlegels. In: Jahrbuch deutscher Bibliophilen und Literaturfreunde (1937). S. 122-126; Schlegel, August Wilhelm: Kritische Ausgabe der Vorlesungen. Bd. 2/I. Hrsg. von Stefan Knödler. Paderborn: Schöningh 2016, S. 10. 3 Katalog der von Aug. Wilh. von Schlegel, Professor an der Königl. Universität zu Bonn, Ritter etc., nachgelaßenen Büchersammlung, welche Montag den 1sten December 1845 und an den folgenden Tagen Abends 5 Uhr präcise bei J. M. Heberle in Bonn öffentlich versteigert und dem Letztbietenden gegen gleich baare Bezahlung verabfolgt wird.

Ә Open Access. (C) 2018 Roger Paulin, publiziert von De Gruyter. (c) BY-Nc-ND Dieses Werk ist lizenziert unter der Creative Commons Attribution-NonCommercial-NoDerivatives 4.0 Lizenz. 
ordnet. Eine systematische Auswertung dieses Katalogs unter Heranziehung des Bücherverzeichnisses von 1811 sowie eine Kollationierung aller Bücherlisten Schlegels wäre ein dringendes Desiderat der Schlegel-Forschung, die aber erhebliche bücherkundige und bibliothekswissenschaftliche Recherchen erfordern würde. Denn beide Sammlungen sind insofern nicht deckungsgleich, als dass im Heberle-Katalog bedeutende Teile von 1811 fehlen, beispielsweise die wichtigen Luther- und Winckelmann-Bestände. Nur Besitzvermerke mit autografischer Widmung wurden bei Heberle verzeichnet (man kannte das Verzeichniß offenbar nicht), während Schlegel in seinem Verzeichniß im Jahr 1811 in mehreren Fällen Herkunft und Schenkungen notiert. Für die intellektuelle Persona Schlegel und seine facettenreichen Vernetzungen sind diese Vermerke von besonderem Interesse.

Dringend notwendig wäre ebenfalls der Vergleich mit der um vieles umfangreicheren Büchersammlung Ludwig Tiecks, 1849-1850 versteigert ${ }^{4}$ und bis vor Kurzem Objekt eines Sonderforschungsprojektes an der Universität Wien, ${ }^{5}$ schon deshalb, weil sowohl Schlegels als auch Tiecks Sammlung ein stark komparatistischer Ansatz zugrunde liegt (Bücherbestände in mindestens zehn Sprachen) und beide ein beweiskräftiges Indiz zur Arbeitsmethode der Autoren liefern, zu ihren ausgeführten Projekten und ebenso zu ihren unausgeführten. Generell lässt sich sagen, dass beide Dichter eine ansehnliche Handbibliothek anlegen; durch Tiecks Bibliophilie wächst die seine ins Hypertrophe (Mindesteinschätzung 9000 Bände), aber auch Schlegel sind solche bibliophilen Anwandlungen nicht fremd. Nachweislich ergänzt Schlegel die eigene Sammlung durch den Gebrauch von öffentlichen und privaten Sammlungen in Genf, Paris, Bern, später Stockholm und Bonn, oder durch den gelehrten Tausch: Er leiht Guillaume Favre in Genf beispielsweise Adelungs Mithridates; ${ }^{6}$ auf Schlegels antiquarische Anfragen hin schreibt Favre: „J'ai feuilleté force bouquins“ („Ich habe eine ganze Menge Bücher durchgestöbert“). ${ }^{7}$

So reizvoll es wäre, anhand von Schlegels Verzeichniß sozusagen den Arbeitstisch des Dichters, Kritikers und Gelehrten zu rekonstruieren, begnüge

4 Vgl. Bibliotheca Tieckiana. Catalogue de la bibliothèque célèbre de M. Ludwig Tieck qui sera vendue à Berlin le 10 décembre 1849 et jours suivants par MM. A. Asher \& Comp. Berlin: Trowitzsch 1849.

5 Vgl. http://complit.univie.ac.at/forschungsprojekte/ludwig-tiecks-library-anatomy-of-a-romantic-and-comparatist-book-collection/ (18.11.2017).

6 August Wilhelm Schlegel an Guillaume Favre. Brief vom 19. Februar 1809. In: Mélanges d'histoire littéraire par Guillaume Favre avec des lettres inédites d'Auguste-Guillaume Schlegel et d'Angelo Mai receuilis par sa famille et publiés par J. Adert. Genf: Ramboz \& Schuchardt 1856, S. LXX.

7 Mscr. Dresd. App. 2712. A11. A2 (1). 
ich mich im Folgenden mit einigen allgemeinen Bemerkungen zu seiner Büchersammlung und richte den Blick gezielt auf einige als Geschenk erhaltene Titel. ${ }^{8}$ Versucht man seine Sammlung annähernd thematisch zu ordnen, so tun sich die Etappen seines Wirkens auf.

Der Vater Johann Adolf Schlegel gibt seinem Sohn seine Sammlung von Bodmer und Breitinger (Nr. 771-775) mit auf den Weg. In Berlin wird Schlegel 1801-1804 den Wert der Schweizer Ästhetik stark relativieren, Bodmers Verdienste als Entdecker der altdeutschen Poesie jedoch würdigen und durch Büchererwerb ergänzen. Aus den Göttinger Jahren ist wenig zu verzeichnen, aber auch diese wenigen sind schon signifikant. Nach Schlegels undankbarer Arbeit an Heynes Virgil-Ausgabe (als, wie er sagt, „Handlanger der Gelehrsamkeit“) ${ }^{9}$ schenkt dieser ihm die Edition (Nr. 189-192), eine verdiente Geste. Immerhin war dies eine nützliche Einübung für Schlegels spätere Sanskrit-Ausgaben in seiner Bonner Zeit. Bürgers Gedichte von 1789 erhält er vom Verfasser (Nr. 443): Es ist die Ausgabe, die Schillers ungnädiger Besprechung zugrunde liegt. Die Bürger'schen Schwerpunkte - vor allem Übersetzungen - werden in den 1790er Jahren sichtbar. In der nächsten Lebensetappe Amsterdam summieren sich die Arbeitspläne zu Shakespeare, zu Dante, vielleicht bereits zur spanischen Literatur, denn „Hofr. Eschenburg“, der ihm die Amsterdamer Hauslehrerstelle zuerst vermittelt hatte, tauscht eine Calderón-Ausgabe aus dem Jahr 1717 gegen ein unbekanntes Buch ein (Nr. 129) - der „Hofr.“ interessierte sich offenbar nicht dafür. ${ }^{10}$ Bei Eschenburg gewinnt Schlegel eine erste Anleitung zur Systematisierung seines in Göttingen erworbenen Wissens, durch den Bücherbesitz wird das bestätigt. Die Konkurrenz mit Eschenburg ist gewiss ein Ansporn zur Shakespeare-Übersetzung. Wilhelm von Humboldt schenkt ihm, wohl in Jena, den Eschenburg'schen Shakespeare (Nr. 193-204), den Schlegel nachweislich mit Aufmerksamkeit studiert. Ob Humboldts Schenkung vor oder nach seinem Brief an Schlegel vom 23. Juli 1796 erfolgte, lässt sich nicht ermitteln. ${ }^{11}$ Sollte Eschenburgs Edition als weiteres Indiz für die nach Humboldts Ermessen letztendliche

8 Die vollständige Liste der Geber umfasst 38 Namen.

9 Indische Bibliothek. Eine Zeitschrift von August Wilhelm von Schlegel. Bonn: Weber 18201830. Bd. 2, S. 5-6.

10 Eschenburgs Sammlung enthielt keinen Calderón. Vgl. Verzeichniß derjenigen Bücher aus dem Nachlasse weil. Herrn Geheime-Justizraths und Professors Dr. Joh. Joachim Eschenburg, welche am 7ten October dieses Jahrs [...] verkauft werden sollen. Braunschweig: Fürstl. Waisenhaus-Buchdruckerei 1822.

11 Vgl. Wilhelm von Humboldt an August Wilhelm Schlegel. Brief vom 23. Juli 1796. In: Klette, Anton: Verzeichnis der von A. W. v. Schlegel hinterlassenen Briefsammlung. Nebst Mittheilung ausgewählter Proben des Briefwechsels mit den Gebrüdern von Humboldt, F. Schleiermacher, B. G. Niebuhr und J. Grimm. Bonn [o. V.] 1868, S. V-VI. 
Unausführbarkeit solcher Übersetzungstätigkeit dienen? Wenn ja, hat sie ihren Zweck verfehlt, denn Eschenburg ist eine brauchbare Stütze für Schlegel und Caroline. Nicht nur das: Wie Eschenburgs eigene Sammlung ist auch Schlegels eine Fundgrube von Übersetzungen jeglicher Art, ein Bekenntnis zu ihrem Wert und ihrer Würde, zur Weltoffenheit und Transferfunktion, die er mit Übersetzungen assoziiert und die er 1827 in seiner Replik an den immer noch skeptischen Humboldt unterstreicht. ${ }^{12}$ Die Wulfila-Bibel, Olearius' Saadi, William Jones’ Manu, Georg Forsters Sakontala (nach William Jones), Hammers Firdusi, Knebels Properz, Schleiermachers Platon, Voss und Gries sowieso, aber auch Frischlins Aristophanes von 1586 bekunden allesamt die kosmopolitische Breite der Übersetzerkunst in Schlegels Verzeichniß.

In Amsterdam beklagt Schlegel seinem Bruder Friedrich gegenüber die dortige Bibliothekslage und die angeblich mangelnden Bestände an italienischer Literatur. So erwirbt er, vermutlich noch in Amsterdam und wohl bei der letzten Auktion im Jahr 1793, ${ }^{13}$ ein paar italienische Schätze aus der berühmten Bolongaro-Crevenna'schen Bibliothek. ${ }^{14}$ Ein Ariost aus dem Jahr 1603 leuchtet ein (Nr. 51), ${ }^{15}$ aber eine Inkunabel aus dem Jahr 1472 (Nr. 137) (,Édition très rare“) ${ }^{16}$ zeugt eher von einer Lust am Büchersammeln. Seine reichhaltige italienische Sammlung enthält überhaupt viele seltene Drucke und weist Vollständigkeit auf - mit Ausnahme des 18. Jahrhunderts, das ihn als neuklassizistisches Zeitalter kaum anspricht -, was ein Projekt zur italienischen Literatur mit Dante im Mittelpunkt als durchaus möglich erscheinen lässt. Solches Ganzheitsdenken wurde durch andere sich aufdrängende Projekte gebremst und blieb eher fragmentarisch; in den Horen, im Athenaeum, in den Jenaer und Berliner Vorlesungen und in den Blumensträußen klingt es aber nach.

Im Gegensatz dazu stehen die englischen Bestände, ja man kann davon ausgehen, dass Schlegel und Caroline die Shakespeare-Übersetzung mithilfe von lediglich zwei annotierten Ausgaben und den Lexika von Johnson und Arnold geleistet haben. Schlegel steht hierin in denkbar starkem Gegensatz zu Eschenburg und Tieck, deren Ausrichtung historisch-kritisch war (,höhere Kritik', wenn man will), Schlegels dagegen rein sprachlich. Seine Ausgaben der Old Plays von Dodsley, von Beaumont und Fletcher oder Ben Jonson wurden vermutlich für die Wiener Vorlesungen erworben, denn dort behandelt er das

\footnotetext{
12 Vgl. Indische Bibliothek (wie Anm. 9), Bd. 2, S. 254-255.

13 Vgl. Dibdin, Thomas Frognall: Bibliomania; or Book Madness. A Bibliographical Romance in Six Parts. London: Longman 1811, S. 106.

14 Es sind insgesamt zehn Titel. Vgl. Catalogue des livres de la bibliothéque [sic!] de M. PierreAntoine Bolongaro-Crevenna. Bd. 3: Belles-Lettres. Amsterdam: Changouin \& Hengst 1789.

15 Vgl. Catalogue (wie Anm. 14), Nr. 4559.

16 Catalogue (wie Anm. 14), Nr. 4564.
} 
englische Drama im Umkreis von Shakespeare etwas ausführlicher. Milton fehlt, die Dichter des 18. Jahrhunderts ebenfalls, dafür besitzt er die Sachautoren Gibbon und Burke. Sein Interesse an der englischen Literatur scheint sich also sehr in Grenzen zu halten.

Besonders aufschlussreich aus Schlegels Jenaer und Berliner Zeit sind die ihm geschenkten Bücher oder solche, deren Drucklegung er betreut. Dass er die Texte seines Freundes Ludwig Tieck aus dessen ,romantischer' Zeit sammelt, ist selbstverständlich. Der leicht chaotische Tieck macht ihm aber seine Minnelieder aus dem Schwäbischen Zeitalter (Nr. 290) zum Geschenk, ein wichtiger Bezugstext für Schlegel als Forscher und Sammler deutscher Altertümer. Inzwischen wissen wir, dass Schlegel die Fahnenkorrektur dieses Buches für seinen bereits nach Ziebingen gezogenen Freund leistete. ${ }^{17}$ Von seinem ebenso fahrigen und säumigen Bruder Friedrich sammelt Schlegel alles. Er erhält immerhin als Gegenleistung das bahnbrechende Über die Sprache und Weisheit der Indier (1808; Nr. 314), an dem sich August Wilhelms eigene Indien-Begeisterung entzündet und das bis heute ein Kerntext der Sanskrit-Rezeption bleibt. Die relativ unbedeutende Corinne-Übersetzung, im Namen Friedrichs, aber eigentlich von Dorothea, aus dem Jahr 1807 (Nr. 270-273) und die Gedichte von 1809 (Nr. 304) kommen hinzu.

Viel dankbarer erweisen sich Schelling und Schleiermacher, die - im Falle Schellings kein Wunder - nie zum engsten Freundeskreis Schlegels gehören, denen er aber aus romantischer Solidarität einen Freundschaftsdienst leistet. Bei Schellings Bruno steht Schlegels Vermerk „,Von diesem Buch habe ich beym Druck die Durchsicht besorgt“ (Nr. 315) und Schelling schenkt ihm mindestens bis 1809 seine neuesten Veröffentlichungen (Nr. 536, 659, 660, 739). Von Schleiermacher erhält er dessen Vertraute Briefe über Friedrich Schlegels Lucinde (Nr. 764), wohl wieder eine Loyalitätsgeste, und er übernimmt die Fahnenkorrektur beim ersten Band von dessen Platon (1804-1807; Nr. 345-348), dem anderen großen Übersetzungswerk der Romantik neben Schlegels eigenem Shakespeare. Auch für Schleiermachers Grundlinien einer Kritik der bisherigen Sittenlehre (1803; Nr. 373) besorgt er den Druck. Ob der Theologe mit diesem Buch den mit Sophie Bernhardi liierten Schlegel beschämen wollte, steht dahin. Der schwedische Legionssekretär Karl Gustav von Brinckmann, der später die erste Begegnung Schlegels mit Madame de Staël inszeniert, muss wohl von des-

17 Ludwig Tieck und die Brüder Schlegel. Briefe. Auf der Grundlage der von Henry Lüdeke besorgten Edition neu hrsg. und kommentiert von Edgar Lohner. München: Winkler 1972, S. 134-135. 
sen Dante-Studien und auch von seiner Sammlerleidenschaft gewusst haben, denn er schenkt ihm eine sehr seltene Divina Commedia aus dem Jahr 1503 (Nr. 134).$^{18}$ Der Berliner neuklassizistische Architekt Hans Christian Genelli, der für die dortige Aufführung von Schlegels verunglücktem Ion das Bühnenbild entworfen hatte, überreicht ihm zum Trost seine Vitruv-Ausgabe (Nr. 54).

Von besonderem Interesse sind - kaum zufällig - die beiden Büchergeschenke Goethes an Schlegel, beide aus der Periode ihrer engsten Beziehung zueinander, der Athenaeums-Zeit. 1798 erhält Schlegel von Goethe sein Hermann und Dorothea (Nr. 1056). Goethe hatte allen Grund, sich gefällig zu erzeigen, denn Schlegel hatte das Epos in der Allgemeinen Literatur-Zeitung überschwänglich gelobt, den Vergleich mit Homer nicht gescheut und seine „vaterländischen" Werte gepriesen. Hinzu kam, dass Goethe - sehr zum Entsetzen einer späteren puristischen Goethe-Philologie - Schlegel gebeten hatte, seine Versepen und längeren Elegien, darunter Hermann und Dorothea, metrisch zu korrigieren. Der Ton ihres Briefwechsels aus dieser Zeit ist freundlich, herzlich sogar, man tauscht Bücher, und Goethe schickt Schlegel die Druckfahnen seiner Propyläen. Ein paar Monate später sendet ihm Schlegel seine Gemälde-Gespräche aus dem Athenaeum, in der Hoffnung, ihm nicht zu missfallen. Goethe schenkt ihm eine Ausgabe der Propyläen (Nr. 369-371), sicher eine huldreiche Geste, vielleicht auch etwas von einem Danaergeschenk, denn die Wendung zur Religion in den Gemälden steht sehr im Gegensatz zur strengen Doktrin der Weimarer Kunstfreunde. Auch wenn die Beziehung zu Goethe (und besonders $\mathrm{zu}$ Schiller) abkühlt, sammelt Schlegel nach wie vor ihre Werke (Wallenstein, Maria Stuart, Farbenlehre).

Schon vor seiner Begegnung mit Madame de Staël und noch als Professor der Poesie und Ästhetik in Jena hatte Schlegel in den Bereichen der altdeutschen Literatur und der iberischen Poesie ansehnliche Sammlungen angelegt. Animiert hat ihn sicher der Wunsch, in Jena wie in Berlin eine Art Kompendium, auf jeden Fall die Grundlinien von einer Geschichte der deutschen und der spanischen Poesie zu präsentieren, was bekanntlich nur in Ansätzen geschehen ist. Selbstverständlich besaß Schlegel die nötigen Hilfsmittel - etwa Bodmer und Breitinger, Eschenburg, Adelung, Erduin Julius Koch, Johann Adolph Nasser -, aber etwas von Tiecks Sammlerleidenschaft scheint ihn geritten zu haben, als er die Inkunabel Der jüngere Titurel (Nr. 49g) sowie mehrere seltene Drucke aus dem 16. und 17. Jahrhundert erwarb. Sein Freund und Protegé Friedrich de la Motte Fouqué unterstützte diese kleine Schwäche, als er Schlegel den Olearius aus dem Jahr 1654 überreichte (Nr. 48). Hierzu ist zu

18 Sie galt im 19. Jahrhundert als Unikat. Vgl. Biografia Dantesca [...] compilata del Sig. Visconte Colomb de Batines. Bd. 1. Prato: Aldina 1845, S. 64. 
sagen, dass man um 1800 auf frühe oder Erstdrucke angewiesen war, bei der spanischen Literatur auf das Sortiment der Buchhändler oder auf den Tausch (beispielsweise Eschenburgs Calderón). Allgemein bleibt festzustellen, dass sich Schlegels deutsche und spanische Bestände durchaus neben Eschenburgs und sogar Tiecks sehen lassen konnten. Nach 1811 wich die Sammlerlust einer eher philologischen Strenge, im Falle des Spanischen hörte nach den Wiener Vorlesungen, in denen Schlegel das spanische Drama ausführlich behandelt hatte, das Sammeln auf.

In Coppet und anderen Wohnsitzen Madame de Staëls war Schlegel nicht ausschließlich auf seine eigene Büchersammlung angewiesen: Er konnte die Staël'sche Bibliothek benutzen. Er erhielt, wie schon erwähnt, Bücher aus Genf. Der bereits genannte Guillaume Favre, der wohl wusste, dass sich Schlegels Interesse an Italien bis zurück auf die Etrusker erstreckte, schenkte ihm Lanzis Saggio di lingua Etrusca (1789; Nr. 727-729). Der befreundete Arzt und Magnetiseur David Ferdinand Koreff übergab ihm eine Ausgabe von Montemayors Diana (Nr. 438). Von Madame de Staëls Freund und Korrespondenten Henri Meister erhielt er dessen Werke über die Unsterblichkeit der Seele (Nr. 637-638). Prosper de Barante, ein Staël-Intimus und Mitglied des innersten Kreises von Coppet, schenkte ihm beide Ausgaben seiner De la littérature française pendant le XVIIIe siècle (Nr. 356, 684). Wohl im Bewusstsein, dass in Coppet die französische Klassik eine Basis der literarischen Gesprächskultur bildete, hat Schlegel - im Gegensatz zu seinem Verfahren mit der entsprechenden englischen Literatur - eine ansehnliche Sammlung von Corneille bis Voltaire angelegt.

Madame de Staëls Büchergeschenke an Schlegel sind daher von besonderem Interesse: Sie zeichnen sich durch ihren Eklektizismus aus. Ein Prachtexemplar von Corinne war wohl angebracht (Nr. 301-302), wo ihr Schlegel doch im Schaffensprozess des Romans unermüdlich beigestanden und ihn durch eine wohlwollende Rezension einem diskriminierenden Leserpublikum in Deutschland bekannt gemacht hatte (eine Auswahl ihrer Werke aus ,l'an IV hatte sie ihm schon verehrt; Nr. 357). Wie er zu einem Don Karlos mit Kupfern stand (Nr. 288), den Staël ihm überreichte, kann man nur vermuten: Seine Wiener Vorlesungen behandeln das Drama eher kritisch als wohlwollend, De l'Allemagne umgekehrt. Staël wusste um Schlegels Leidenschaften und Faibles: Ein Exemplar von Villoisons epochemachender Homer-Ausgabe (1788) kam sicher gut an (Nr. 9-12), wenngleich Schlegels Homer-Phase weitgehend vorüber war. Für Lombardis Dante-Ausgabe aus dem Jahr 1791 trifft das ebenfalls zu (Nr. 100-102). Eine Ausgabe von Vasari (1767) war für den Kunstbeflissenen immer von Nutzen (Nr. 146-152). Nicht Madame de Staël selbst, sondern ihr Sohn Auguste hat Schlegel Baillys Histoire de l'Astronomie (in einer Ausgabe von 
1805) geschenkt (Nr. 307-308), ein Kerntext für Schlegels Ausführungen zu den sprachlichen, kulturellen und technischen Ursprüngen der Menschheit, von seinen unveröffentlichten Considérations sur la civilisation en général (1805) bis hin zu seinem späteren Aufsatz über den Zodiacus. Augustes Geste - er war wohl noch unter zwanzig - ist ein weiteres Zeugnis für das gute Einvernehmen zwischen Schlegel und der Familie Staël, das bis zum Tod aller Staël-Kinder andauerte.

„Sey und bleibe nur ja überall und in allem ein Deutscher“, hatte ihm sein Bruder Friedrich in die Diaspora hineingerufen. ${ }^{19}$ Es war die mehrmals artikulierte Befürchtung, Schlegel könnte im französischen Kulturambiente Madame de Staëls seinem ,Vaterland' untreu werden. Solche Besorgnisse waren unnötig, denn im Schweizer Exil machte Schlegel aus der Not eine Tugend und legte eine ansehnliche Sammlung über Topografie, Geschichte, Folklore und Sprache der deutschsprachigen Schweiz an, von Tschudi bis Johannes von Müller, ja überhaupt zur Länderkunde Deutschlands. Schlegels eigene Umriße, entworfen auf einer Reise durch die Schweiz gehören hierher. Von dem Berner Oberbibliothekar Johann Rudolf Wyss, dem Herausgeber der Zeitschrift Alpenrosen, in der diese Reiseeindrücke erschienen, erhielt Schlegel dessen Vorlesungen über das höchste Gut (1811; Nr. 644). Friedrich de la Motte Fouqué, Schlegels Schützling und der Begründer der altdeutsch-ritterlichen Richtung in Drama und Erzählung der Romantik, schickte ihm seine vaterländischen Ergüsse Sigurd, der Schlangentödter (Nr. 160), Eginhard und Emma (Nr. 543) und wie sie sonst alle heißen.

Einen symbolischen Stellenwert in Schlegels Büchersammlung hat die von Franz von Baader überreichte Schrift Beiträge zur dinamischen Philosophie im Gegensaze der mechanischen (1809; Nr. 539). Diese Aufsatzsammlung Baaders, weitgehend eine Auseinandersetzung mit Kant, berührt sich mit der mystischtheosophischen Wende in Coppet, die schon vor Schlegels Ankunft eingesetzt hatte, spätestens bis 1810 andauerte und die eine lange Passage in De l'Allemagne, „De la disposition religieuse appelée mysticité“, inspiriert hatte. Die Aufenthalte in Coppet zweier Quasimystiker, Zacharias Werner und Frau von Krüdener, gehören auch hierher, Karl Viktor von Bonstettens abwertendes Urteil ebenfalls: „[D]ie Leute werden alle noch katholisch, böhmisch, martinistisch, mystisch, alles durch S[chlegel]““ ${ }^{20}$ Schlegel besaß alle bis dahin erschie-

19 Friedrich Schlegel an August Wilhelm Schlegel. Brief vom 8. Juni 1813. In: Friedrich Schlegels Briefe an seinen Bruder August Wilhelm. Hrsg. von Oskar Walzel. Berlin: Speyer \& Peters 1890. S. 541-544, hier S. 542.

20 Karl Viktor von Bonstetten an Friederike Brun. Brief vom 12. Oktober 1809. In: Bonstettiana. Briefkorrespondenzen Karl Viktor von Bonstettens und seines Kreises. Teilband X/2. Hrsg. von Doris u. Peter Walser-Wilhelm. Göttingen: Wallstein 2003. S. 653-656, hier S. 654. 
nenen Dramen Werners und stand ihm eine Zeitlang näher als seinem wenig verlässlichen Freund Ludwig Tieck. Ihre Gespräche reflektieren beider Lektüre von Jakob Böhme und Louis-Claude de Saint-Martin, der Böhme ins Französische übersetzt hatte. Schlegel schaffte eine entsprechende Büchersammlung an: Böhme und Saint-Martin selbstverständlich, Madame de Guyon, Thomas à Kempis, weiterhin Friedrich Leopold von Stolbergs Geschichte der Religion Jesu Christi, das die Kreise von Münster und Coppet sowie deren mystischen Quietismus miteinander verbindet. Während Staëls mystische Anwandlungen ihren protestantischen Glauben nie ernsthaft erschütterten, zeugt Schlegels zeitweiliges Bekenntnis zur theologischen Gegenaufklärung von einer radikalen Abwendung von der lutherischen Orthodoxie seines Elternhauses. 